\title{
The effects of a task-irrelevant visual event on spatial working memory
}

\author{
Stefan Van der Stigchel, Hanneke Merten, Martijn Meeter, and Jan Theeuwes \\ Vrije Universiteit Amsterdam, Amsterdam, The Netherlands
}

\begin{abstract}
In the present experiment, we investigated whether the memory of a location is affected by the occurrence of an irrelevant visual event. Participants had to memorize the location of a dot. During the retention interval, a task-irrelevant stimulus was presented with abrupt onset somewhere in the visual field. Results showed that the spatial memory representation was affected by the occurrence of the external irrelevant event relative to a control condition in which there was no external event. Specifically, the memorized location was shifted toward the location of the task-irrelevant stimulus. This effect was only present when the onset was close in space to the memory representation. These findings suggest that the "internal" spatial map used for keeping a location in spatial working memory and the "external" spatial map that is affected by exogenous events in the outside world are either the same or tightly linked.
\end{abstract}

In everyday life, we constantly perform tasks for which it is necessary to keep relevant information temporarily in memory. It is thought that this information is kept active in a system referred to as working memory. Working memory was described by Baddeley (1986) as "a system for the temporary maintenance and manipulation of information, necessary for the performance of such complex cognitive activities as comprehension, learning and reasoning" (p. 34). Baddeley and Hitch (1974) proposed a multiple-component model of working memory consisting of a "central executive" and two "slave systems": the phonological loop and the visuospatial sketchpad. These slave systems are specialized in the processing and temporary storage of verbal and spatial information, respectively.

The visuospatial sketchpad is less well understood than the phonological loop. Recent research suggests a strong link between spatial attention and visuospatial working memory (Awh \& Jonides, 2001; Cowan et al., 2005; Engle, 2002; Postle, 2006). For instance, it is proposed that spatial rehearsal in working memory is accomplished through shifts of covert attention toward the memorized location. Two findings from Awh, Jonides, and ReuterLorenz (1998) support this hypothesis. First, when a location was held in spatial working memory, processing of stimuli was more efficient at that location than at other locations. Such benefits are similar to those typically found when the location is attended (Posner, 1980). Second, Awh et al. (1998) showed that when the task required a shift in spatial attention away from the memorized location, spatial working memory accuracy was impaired. Brain imaging studies have also suggested a link between attention and spatial working memory (Awh et al., 1999;
Postle, Awh, Jonides, Smith, \& D'Esposito, 1999). It was shown that spatial rehearsal led to increased activation in the early visual areas contralateral to the memorized location, similar to what is observed in tasks in which spatial attention is directed to a location.

Recently, evidence was also provided for a link between visuospatial working memory and the low-level eye movement system. Just as the eyes may deviate away from visible stimuli (Van der Stigchel, Meeter, \& Theeuwes, 2006), Theeuwes, Olivers, and Chizk (2005) showed that eye movement trajectories also deviate away from remembered stimuli (see also Theeuwes, Van der Stigchel, \& Olivers, 2006). Their experiment consisted of two conditions: one in which the location of an onset had to be remembered, and one in which the onset was irrelevant. After the onset disappeared, an arrow pointing up or down from the central location indicated the direction in which a saccade had to be made. Results showed that the eye movement deviation away from the dot was stronger in the condition in which the participants had to remember the location of the onset.

On the basis of these studies, it can be concluded that there is a strong link between spatial working memory and the attentional and oculomotor system. Given the known effects of task-irrelevant stimuli on both the attentional and the oculomotor systems, the present study investigated the effect of task-irrelevant stimuli on spatial working memory representations. It is well-known that visual transients can capture attention in an exogenous way (Theeuwes, 1994; Yantis \& Jonides, 1984), even when they are uninformative and participants are instructed to ignore them (Jonides, 1981; Remington, Johnston, \& Yantis, 1992). With respect to eye movements, task-irrelevant

S.Van der Stigchel, s.vanderstigchel@uu.nl 
onsets are known to increase saccade latencies (Walker, Deubel, Schneider, \& Findlay, 1997), influence saccade trajectories (Van der Stigchel et al., 2006), and capture the eyes (Theeuwes, Kramer, Hahn, \& Irwin, 1998). Whereas it has been shown that nonpredictive onsets enhance the transfer of perceptual representations into working memory (Schmidt, Vogel, Woodman, \& Luck, 2002), it is unknown what happens to the spatial memory representation of a location when an onset is presented. Previous studies have indicated that spatial memory representations can drift because of various factors. For instance, locations stored in spatial working memory can drift toward locations stored in long-term memory or toward previously experienced prototypes (Spencer \& Hund, 2002, 2003; Spencer, Smith, \& Thelen, 2001). Similar drifts might also be observed in the presence of an external visual event like a task-irrelevant onset.

In our experiment, participants were asked to remember a target location during a retention interval. In order to examine the effect of a task-irrelevant onset on the representation of that location in spatial working memory, we investigated whether the reported memory location (as indicated by a mouse click) was affected by the taskirrelevant stimulus. In the present experiment, the task of participants was to memorize a target location. An abrupt onset was presented after a variable interval during the retention interval. We presented the onset after a 400- to 600 -msec retention interval to ensure that any effects of the onset would be on the memory representation and not occur during encoding of the target location. By comparing trials with and without an onset, we determined whether the reported memory location shifted with respect to the location of the onset. More specifically, we determined whether the location kept in memory shifted toward or away from the external stimulus event. There are two possible outcomes. One outcome could be an attraction toward the abrupt onset, resulting in a shift of the memory representation toward the onset location. Another outcome could be a shift of the memory location away from the onset location. This could be caused by the active inhibition of the activity of the abrupt onset, or by the lateral inhibition of the memory location due to a shift of spatial attention to the onset location. Both of these options would result in a shift in activity away from the onset location.

\section{METHOD}

\section{Participants}

Ten students were paid for their participation and reported having normal or corrected-to-normal vision. They were not familiar with the purpose of the experiment.

\section{Stimuli}

Figure 1 illustrates the display sequence. At the start of each trial, participants viewed a display with a central fixation point on a black background. After $500 \mathrm{msec}$, the target appeared, a light gray cross $\left(1.0^{\circ}\right.$ of visual angle; luminance $\left.10.3 \mathrm{~cd} / \mathrm{m}^{2}\right)$, in one of the four quadrants. The target was visible for $500 \mathrm{msec}$. For each participant, two out of nine possible locations in each quadrant were randomly assigned. For each quadrant, targets appeared at one of these two locations. The nine possible locations in each quadrant were located in a $3 \times 3$ grid (measuring $4.2^{\circ} \times 4.2^{\circ}$ ). The grid was centered on $x=$ $\pm 5.9^{\circ}, y= \pm 3.7^{\circ}$ from the fixation point.

In the onset condition, an abrupt onset appeared after a variable interval of 400-600 msec after the target was switched off. The onset was a light gray dot $\left(1.0^{\circ}\right.$ of visual angle; luminance $\left.10.3 \mathrm{~cd} / \mathrm{m}^{2}\right)$ and was visible for $100 \mathrm{msec}$. The onset was presented in $75 \%$ of the trials. The onset could appear equally often in any of the four quadrants and appeared in the same quadrant as the target in $25 \%$ of the trials. If the onset was presented in the same quadrant as the target, the distance of the onset to the target location was kept constant at $1.67^{\circ}$, and the onset was presented either at the top left, top right, bottom left, or bottom right of the target location. If the onset was presented in a different quadrant, the location of the onset was randomly chosen, although at least $3.0^{\circ}$ away from the target location. After a total retention interval of $1,500 \mathrm{msec}$, the mouse cursor became visible, which indicated that a response could be made.

\section{Procedure and Design}

Each participant was tested in a dimly lit room. Their head rested on a chinrest, located $75 \mathrm{~cm}$ away from the monitor. Eye movements were recorded by means of an Eyelink tracker with a $500-\mathrm{Hz}$ temporal resolution and a $0.1^{\circ}$ spatial resolution. The participants had to perform a memory task. They were instructed to memorize the location of the target as accurately as possible. They were also told to ignore abrupt onsets if they appeared and to execute a mouse click on the location they believed to be the location of the target. It was made clear to the participants that they had to keep their eyes fixated on the fixation point until the mouse pointer became visible. Participants performed 32 practice trials and 512 experimental trials. After each trial, the participants received feedback about their

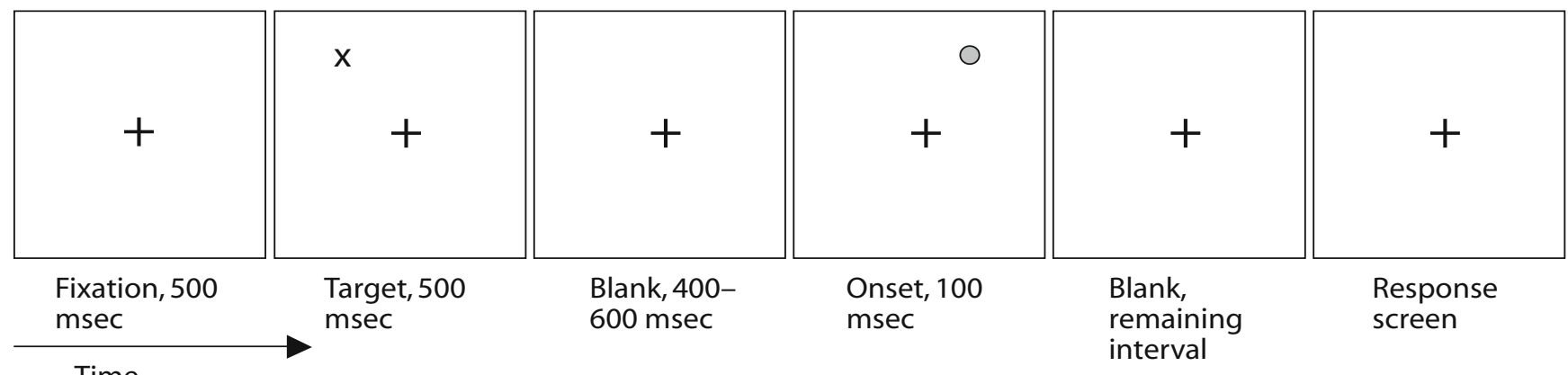

Figure 1. Example of the display sequence (not drawn to scale). Participants had to remember the target location. In the onset condition an irrelevant stimulus appeared after a variable interval. This stimulus did not appear in the no-onset condition. The total retention interval for both conditions was 1,500 msec. 
performance; the target location and the location of the mouse click were shown.

\section{RESULTS}

\section{Discarded Trials}

Trials in which participants did not fixate well were discarded. This was defined as trials on which the eyes were not within $2.5^{\circ}$ of visual angle of the fixation cross from the start of the trial until the mouse cursor became visible. We also removed outliers from the analyses: If the distance between the target location and the location of the mouse click exceeded 2.5 standard deviations from the mean distance, the trial was discarded. The total loss of trials was $7.2 \%$.

\section{No-Onset Trials}

On the no-onset trials, participants had the tendency to undershoot the location of the target $[t(9)=5.80, p<$ $.001]$. The distance between the target and central fixation $\left(M=7.10^{\circ}, S E=0.22^{\circ}\right)$ was larger than the distance between the mouse click and central fixation $\left(M=6.87^{\circ}\right.$, $S E=0.22^{\circ}$ ).

\section{Deviation From Target}

To determine whether there was a general interference effect of the onset, the mean deviation between the mouse click and the target location was computed for each quadrant (see Table 1). An ANOVA with condition (no onset, onset in same quadrant, onset in different quadrant) as a factor showed no difference between the three conditions $(F<1)$. The mean deviation was $0.73^{\circ}\left(S E=0.06^{\circ}\right)$.

\section{Vector Comparison}

We compared trials with and without onsets having the exact same memorized location. For each trial with an onset, we subtracted from the reported memorized location the average reported memorized location on trials with no onset. This was done because participants tended to undershoot the target location on trials without an onset. The resulting vector ("change vector" in Figure 2) was decomposed into two components: one the vector

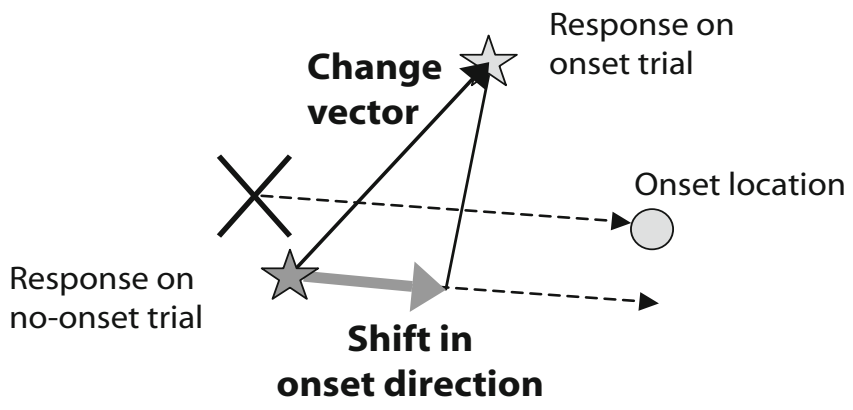

Figure 2. An illustration of the vector comparison computation used to investigate the effect of an abrupt onset on the spatial memory representation. The decomposition on the target-onset vector ("shift in onset direction") was used as the dependent measure. " $X$ " represents the true target location.
Table 1

Summary of the Results

\begin{tabular}{|c|c|c|c|c|c|c|}
\hline & \multicolumn{6}{|c|}{ Onset } \\
\hline & \multicolumn{2}{|c|}{ None } & \multicolumn{2}{|c|}{$\begin{array}{l}\text { In Same } \\
\text { Quadrant }\end{array}$} & \multicolumn{2}{|c|}{$\begin{array}{c}\text { In Different } \\
\text { Quadrant }\end{array}$} \\
\hline & $M$ & $S E$ & $M$ & $S E$ & $M$ & $S E$ \\
\hline Deviation from target & $0.73^{\circ}$ & $0.05^{\circ}$ & $0.73^{\circ}$ & $0.06^{\circ}$ & $0.74^{\circ}$ & $0.06^{\circ}$ \\
\hline Vector comparison & & & $0.153^{\circ}$ & $0.052^{\circ}$ & $0.028^{\circ}$ & $0.035^{\circ}$ \\
\hline
\end{tabular}

from target to onset, ${ }^{1}$ and one the orthogonal vector. The decomposition on the target-onset vector ("shift in onset direction" in Figure 2) was used as a measure of the shift in memorized location due to the onset. Positive values in the results signify a shift in the direction of the onset, negative values a shift in the opposite direction.

The mean shift in the onset direction was indeed positive $[F(1,9)=5.96, p<.05]$. This shift was $0.125^{\circ}$ larger when the onset was presented in the same quadrant than when it was presented in a different quadrant $[F(1,9)=$ $6.38, p<.04$; see Table 1]. We then determined for both conditions separately whether the shift was different from zero. When the onset was presented in the same quadrant, there was a large shift in the direction of the onset $[t(9)=$ $2.95, p<.02]$. However, this effect was absent when the onset was presented in a different quadrant from the target $[t(9)=0.78, p>.40]$.

To investigate whether there was an effect of the exact onset location when the onset was presented in the same quadrant as the target, we collapsed over quadrants and investigated the effect of onset location with respect to the distance from central fixation. Onsets could be farther away from fixation than the target location (remote onsets), closer to fixation than the target location (close onsets), or at approximately the same distance from fixation (to the upper left or bottom right of the target location in Figure 3). An ANOVA with distance from fixation as factor revealed a main effect of distance $[F(2,18)=7.63, p<$ .01]. Post hoc comparisons showed that a remote onset location evoked a larger shift toward itself than did onsets at other distances from fixation $[F(1,9)=11.46, p<.01]$. This effect can be seen in a Figure 3 , which shows a scatterplot of the single-trial data and mean responses for the no-onset condition and the different onset locations.

\section{DISCUSSION}

The present study shows that spatial memory representations are affected by the occurrence of an external visual event. Participants had to memorize a target location for a fixed period. During this period, a task-irrelevant stimulus was presented with abrupt onset in the same or in a different quadrant from the memory location. We presented the onset sufficiently long after the probe to exclude the possibility that the onset interfered with the encoding of the probe. When compared with trials without an onset, the location kept in memory shifted toward the external stimulus event on trials in which the onset was presented. This effect was only present when the external event was close in space to the memory representation. It was es- 

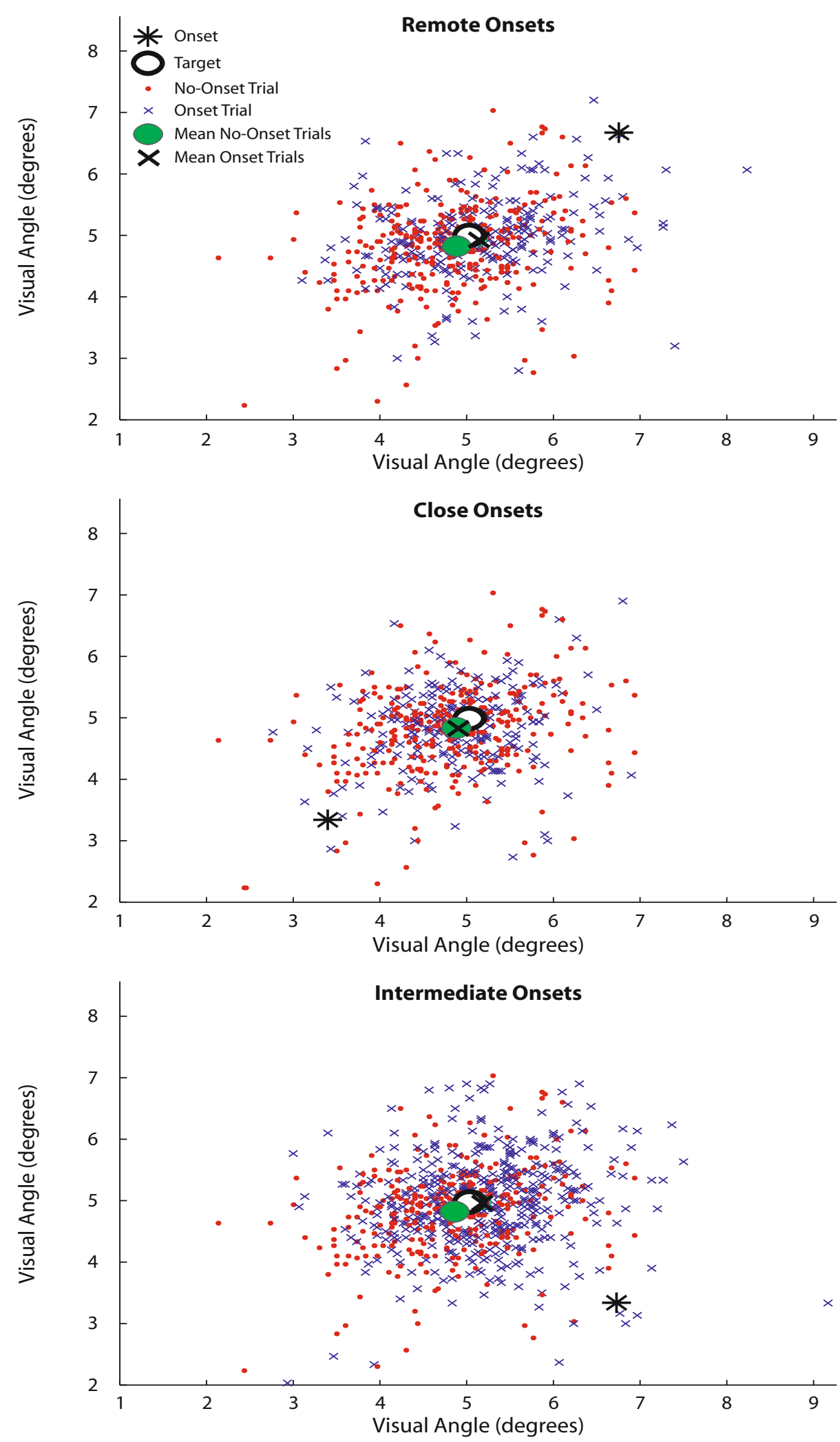

Figure 3. A scatterplot of the single-trial data for the trials in which the irrelevant stimulus appeared in the same quadrant as the target. Separate plots are presented for the different distances of the onset from fixation. Responses were collapsed across the four quadrants to the top right part of the computer screen, making fixation on the bottom left of the figure. Responses were normalized to one target location. Besides showing the target and the onset locations, the figure further shows the mean responses for the no-onset condition and for the three onset locations. It can be seen that the shift of the memory location is especially pronounced for the remote onset. 
pecially pronounced when the onset was presented at a remote point from fixation.

There are a number of possible explanations for the effects of the task-irrelevant stimulus. Since it is known from previous research that visual transients capture spatial attention in an exogenous way (Theeuwes, 1994; Yantis \& Jonides, 1984), the capture of attention by the onset could have interfered with the memory representation of the target. The capture of attention would have evoked a shift of attention away from the memory location and shifted the representation of the target in visuospatial working memory in the direction of the onset. This would be in line with the idea that there is a close link between visuospatial working memory and spatial attention (Awh \& Jonides, 2001; Cowan et al., 2005; Engle, 2002; Postle, 2006). It must be noted that we do not have a direct measure of whether the onset actually captured attention. Moreover, even if there had been evidence that the onset captured attention, one could still question whether this capture of attention caused the shift of the memory location.

There are several other explanations that cannot be excluded. It is possible that the task-irrelevant stimulus actually replaced the target in memory on a subset of trials, which would result in a shift of the mean reported location in the direction of the onset. This would result in two distributions of responses, namely on the target and the onset location. Scatterplots of the mouse clicks, however, do not show these two distributions, but point to one single distribution around the target location, making this explanation unlikely.

One could also argue that the present observation is an effect on the motor system instead of on spatial working memory. It could be that participants programmed the motor response immediately upon seeing the target and retained this motor program during the retention interval. The onset could have distorted the motor system in such a way that it biased the response in the direction of the onset without influencing the perceptual memory of the target location. However, given the evidence for a close link between the memory system and both the attentional and motor systems (Awh \& Jonides, 2001; Cowan et al., 2005; Engle, 2002; Postle, 2006; Theeuwes et al., 2005; Theeuwes et al., 2006), there might be little functional difference between a memory and a motor effect.

On the basis of the present results, we hypothesize that the spatial memory representation and the task-irrelevant stimulus are represented on a common spatial map. In order to remember the location of a target, some activity needs to be maintained in the map at a location corresponding to that of the target. This might occur by attending to the location of the target in absence of visual information (Awh et al., 1998), but that is not crucial for the argument. When, in the present experiment, an abrupt onset was presented, the exogenous activity that this onset causes a shift within the memory representation. These findings suggest that the "internal" spatial map, used for keeping a location in spatial working memory, and the "external" spatial map that is affected by exogenous events in the outside world are either the same or tightly linked. The present results further show that the memory representation was only af- fected when the memory and onset locations were closely aligned. This suggests that a shift in memory representation requires that the neuronal populations involved in coding for the memory and for the onset overlap. When an onset is remote from the memory location, there is no overlap between the populations coding both locations, and the memory representation is unaffected.

Moreover, when the distance between the memory location and the onset was small, the effect was most pronounced when the onset was presented remote from central fixation. It might be that remote locations are coded by larger neuronal populations than are close locations, as has for instance been found in the superior colliculus, a low-level eye movement area (Soetedjo, Kaneko, \& Fuchs, 2002). If this is true, the overlap between the populations is larger for remote onsets than for close onsets. This also explains why we did not observe a general interference effect of the onset as measured by the mean deviation between the mouse click and the target location. Because participants tended to undershoot the target in the no-onset condition, a shift in the direction of the remote onset resulted in an "overshoot" effect, keeping the absolute distance to the target constant.

Modifications in memory representations might originate from the lateral intraparietal sulcus (LIP). Indeed, neurons in LIP are active when a location is held in memory. This was shown in an experiment in which monkeys executed a saccade to a memorized location (Bisley \& Goldberg, 2003). During the time that the monkey held the location in memory, LIP neurons coding for the target location were active. A similar mechanism might be at play here. The initial short-lived automatic activation caused by the target presentation is taken over by more sustained voluntary activation. When an abrupt onset is presented, this causes activation in the LIP and shifts the target representation in the direction of this new peak of activity.

In contrast to the attraction effects observed here, repulsion effects were observed in recent eye movement studies from our lab that revealed that keeping a location in memory influences the oculomotor system (Theeuwes et al., 2005; Theeuwes et al., 2006). These studies showed that eye movements deviated away from the memory location, pointing to repulsion from the memory location. However, in these studies a motor response had to be made to a location different from the memory location. Because it is hypothesized that the location of a spatial memory representation is continuously activated, a motor response to a different location in space requires the active suppression of the motor response toward the memory location, leading to repulsion of this movement from the memory location. In contrast, in the present experiment, the onset location was task-irrelevant ${ }^{2}$ and therefore not activated during the initiation of the motor response toward the memory location. This made active suppression unnecessary.

To conclude, we have shown that an exogenous event in the "outside world" can affect a mental memory representation in the "internal world." An exogenous abrupt onset caused a shift in the memory representation in the direction of the onset location. 


\section{AUTHOR NOTE}

This research benefited from NWO (Netherlands Organization for Scientific Research) Grant 402-01-630-PROG to J.T. and from an NWO VENI Grant to M.M. Correspondence concerning this article should be addressed to S. Van der Stigchel, Experimental Psychology, Helmholtz Institute, Utrecht University, Heidelberglaan 2, 3584 CS, Utrecht, The Netherlands (e-mail: s.vanderstigchel@uu.nl).

\section{REFERENCES}

Awh, E., \& JoNides, J. (2001). Overlapping mechanisms of attention and spatial working memory. Trends in Cognitive Sciences, 5, 119. 126.

Awh, E., Jonides, J., \& Reuter-Lorenz, P. A. (1998). Rehearsal in spatial working memory. Journal of Experimental Psychology: Human Perception \& Performance, 24, 780-790.

Awh, E., Jonides, J., Smith, E. E., Buxton, R. B., Frank, L. R. Love, T., ET AL. (1999). Rehearsal in spatial working memory: Evidence from neuroimaging. Psychological Science, 10, 433-437.

BAdDeley, A. D. (1986). Working memory. Cambridge, MA: MIT Press.

BadDeley, A. D., \& Hitch, G. (1974). Working memory. In G. Bower (Ed.), The psychology of learning and motivation (Vol. 8, pp. 47-89). New York: Academic Press.

Bisley, J. W., \& GoldberG, M. E. (2003). Neuronal activity in the lateral intraparietal area and spatial attention. Science, 299, 81-86.

Cowan, N., Elliott, E. M., Saults, J. S., Morey, C. C., Mattox, S., Hismuatullina, A., ET Al. (2005). On the capacity of attention: Its estimation and its role in working memory and cognitive aptitudes. Cognitive Psychology, 51, 42-100.

ENGLE, R. W. (2002). Working memory capacity as executive attention. Current Directions in Psychological Science, 11, 19-23.

JoNIDES, J. (1981). Voluntary versus automatic control over the mind's eye's movement. In J. Long \& A. Baddeley (Eds.), Attention and performance IX (pp. 187-203). Hillsdale, NJ: Erlbaum.

Posner, M. I. (1980). Orienting of attention: The VIIth Sir Frederic Bartlett Lecture. Quarterly Journal of Experimental Psychology, 32, 3-25.

Postle, B. R. (2006). Working memory as an emergent property of the mind and brain. Neuroscience, 130, 23-38.

Postle, B. R., Awh, E., Jonides, J., Smith, E. E., \& D’Esposito, M. (1999). The where and how of attention-based rehearsal in spatial working memory. Cognitive Brain Research, 20, 194-205.

Remington, R. W., Johnston, J. C., \& Yantis, S. (1992). Involuntary attentional capture by abrupt onsets. Perception \& Psychophysics, 51, 279-290.

Schmidt, B. K., Vogel, E. K., Woodman, G. F., \& LucK, S. J. (2002). Voluntary and automatic attentional control of visual working memory. Perception \& Psychophysics, 64, 754-763.
Soetedjo, R., Kaneko, C. R. S., \& Fuchs, A. F. (2002). Evidence against a moving hill in the superior colliculus during saccadic eye movements in the monkey. Journal of Neurophysiology, 87, 27782789.

SPencer, J. P., \& Hund, A. M. (2002). Prototypes and particulars: Geometric and experience-dependent spatial categories. Journal of Experimental Psychology: General, 131, 16-37.

Spencer, J. P., \& Hund, A. M. (2003). Developmental continuity in the processes that underlie spatial recall. Cognitive Psychology, 47, 432-480.

Spencer, J. P., Smith, L. B., \& Thelen, E. (2001). Tests of a dynamic systems account of the A-not-B error: The influence of prior experience on the spatial memory abilities of 2-year-olds. Child Development, 72, 1327-1346.

Theeuwes, J. (1994). Stimulus-driven capture and attentional set: Selective search for color and visual abrupt onsets. Journal of Experimental Psychology: Human Perception \& Performance, 20, 799-806.

Theeuwes, J., Chizk, C., \& Olivers, C. N. L. (2005). Remembering a location makes the eyes curve away. Psychological Science, 16, 196-199.

Theeuwes, J., Kramer, A. F., Hahn, S., \& Irwin, D. E. (1998). Our eyes do not always go where we want them to go: Capture of eyes by new objects. Psychological Science, 9, 379-385.

Theeuwes, J., Van der Stigchel, S., \& Olivers, C. N. L. (2006). Spatial working memory and inhibition of return. Psychonomic Bulletin \& Review, 13, 608-613.

Van der Stigchel, S., Meeter, M., \& Theeuwes, J. (2006). Eye movement trajectories and what they tell us. Neuroscience \& Biobehavioral Reviews, 30, 666-679.

Walker, R., Deubel, H., Schneider, W. X., \& Findlay, J. M. (1997). Effect of remote distractors on saccade programming: Evidence for an extended fixation zone. Journal of Neurophysiology, 78, 1108-1119.

YANTIS, S., \& Jonides, J. (1984). Abrupt visual onsets and selective attention: Evidence from visual search. Journal of Experimental Psychology: Human Perception \& Performance, 10, 601-621.

\section{NOTES}

1. Using the dot product between the change vector and the targetdistractor vector, divided by the length of the target-distractor vector.

2. It should be noted that there is a possibility that participants noticed that the target was always presented at a fixed distance from the onset when they appeared in the same quadrant. This would make the onset somewhat task relevant. However, if this were true, one would expect the participants to perform better when the onset appeared in the same quadrant as the target. Such an effect was not found.

(Manuscript received December 4, 2006; revision accepted for publication March 13, 2007.) 